Muséologies

Les cahiers d'études supérieures

muséologies

\title{
Quand loisirs et éducation scientifique font bon ménage au Palais de la découverte
}

\section{Jack Guichard}

Volume 3, numéro 2, printemps 2009

URI : https://id.erudit.org/iderudit/1033572ar

DOI : https://doi.org/10.7202/1033572ar

Aller au sommaire du numéro

Éditeur(s)

Association Québécoise de Promotion des Recherches Étudiantes en

Muséologie (AQPREM)

ISSN

1718-5181 (imprimé)

1929-7815 (numérique)

Découvrir la revue

Citer cet article

Guichard, J. (2009). Quand loisirs et éducation scientifique font bon ménage au Palais de la découverte. Muséologies, 3(2), 182-191.

https://doi.org/10.7202/1033572ar d'utilisation que vous pouvez consulter en ligne.

https://apropos.erudit.org/fr/usagers/politique-dutilisation/ 


\section{Article treize}

\section{Quand loisirs et éducation scientifique}

\section{font bon ménage au Palais de la découverte}

JACK GUICHARD, PROFESSEUR DES UNIVERSITÉS, EST LE DIRECTEUR DU PALAIS DE LA DÉCOUVERTE À PARIS. IL EST AUSSI ENSEIGNANT ET CHERCHEUR À L'ECOLE DOCTORALE «SCIENCES PRATIQUES* DE LÉCOLE NORMALE SUPÉRIEURE DE CACHAN. IL A FAIT PARTIE DE L'ÉQUIPE QUI EN 1980 A ENGAGÉ LE PROJET DE LA CITÉ DES SCIENCES ET DE LINDUSTRIE DE LA VILLETTE IL Y A ÉTÉ RESPONSABLE SCIENTIFIQUE DE PLUSIEURS EXPOSITIONS POUR LES JEUNES, EN PARTICULIER LA CITÉ DES ENFANTS. IL A DIRIGEE DE NOMBREUX TRAVAUX DE RECHERCHE SUR LA VULGARISATION ET LÉDUCATION SCEENTIFIQUE ET A PUBLIE DES OUVRAGES SUR CES THEMES, PAR EXEMPLE MÉDIATIOUE DES SCIENCES (PARIS:PRESSES UNIVERSITAIRES DE FRANCE) EN2000. [guichard@mac.com] 
Le contexte et les objectifs de l'école sont très différents, voire opposés à ceux de la visite de loisir d'un musée de science. Pourtant il est possible de faire se rejoindre dans un même lieu ces deux approches, cette stratégie ayant d'ailleurs pour intérêt de développer l'intérêt des jeunes pour les sciences, de fournir des supports pédagogiques aux enseignants et de développer les visites de loisir. C'est la gageure que réussit le Palais de la découverte à Paris depuis 70 ans, et encore plus aujourd'hui.

Pour introduire ce propos, nous raconterons une anecdote. Lors de la dernière exposition sur les dinosaures présentée au Palais de la découverte, beaucoup de visiteurs sont venus pour ce sujet attractif, sans savoir qu'ils venaient dans un musée de science. Certains disaient qu'ils ne se seraient pas déplacés s'ils l'avaient su, car les sciences, trop difficiles, leur faisaient peur. L'organisation des expositions les a amenés à passer dans des expositions plus scientifiques, comme celle sur la lumière, qui les ont intéressés et qui leur ont donné envie de revenir. 


\section{Le musée n'est pas l'école}

Bien que ce soit une évidence que le musée n'est pas l'école, il est nécessaire de bien analyser les différences entre ces deux institutions afin de faire émerger ce qui les rapproche. L'école est un lieu de travail qui répond à une obligation scolaire d'éducation, alors que le musée est d'abord un lieu de loisir dans un but de culture. L'école est contrainte par des programmes, alors que le musée est libre de ces choix thématiques. L'éducation scolaire a l'avantage de se construire dans une progression sur plusieurs années, alors que la visite du musée est un moment fort mais ponctuel. L'école a l'avantage de toucher des publics homogènes du point de vue de l'âge et des savoirs, alors que l'exposition doit toucher des publics variés et hétérogènes. Enfin l'école touche des publics captifs, alors que le musée doit captiver ses publics!

En dépit de ces différences, l'école peut apporter au musée, et vice versa. L'école doit donner envie aux enfants d'aller au musée et le musée doit aider l'école en sensibilisant les élèves pour leur donner le goût des sciences.

L'exposition scientifique peut faire vivre aux enfants une immersion avec des expériences et des objets spectaculaires, déclenchant une émotion qui marque la mémoire. Elle peut faire vivre des situations de découverte qui suscitent le questionnement. L'école quant à elle peut ensuite mettre en place des démarches d'investigation dans la durée. En outre, des expositions comme celles du Palais de la découverte présentent des expériences et des phénomènes en vraie grandeur, expériences que les élèves ne connaissaient que théoriquement par les schémas des livres.

Le Palais de la découverte expose la science en train de se faire, ce qui peut faire évoluer les programmes scolaires, en particulier en testant et en développant du matériel pédagogique qui illustre de nouveaux concepts scientifiques. Ce contact avec des scientifiques et la possibilité de faire des expériences sont aussi très importants pour le développement de vocations scientifiques.

Des lieux pour développer des vocations scientifiques chez les jeunes

Les musées scientifiques, comme le Palais de la découverte, sont des lieux de découverte qui ont un effet positif sur le déclenchement des vocations scientifiques. Une enquête effectuée en 2006 auprès de plus de 300 scientifiques français (ingénieurs, chercheurs, enseignants) a montrél'influence de la visite de musées scientifiques sur leur vocation. Le Palais de la découverte, dû à son âge (70 ans), a eu un rôle sur la vocation de $60 \%$ des scientifiques 
parisiens de plus de 35 ans, la Cité des sciences sur $20 \%$ des moins de 35 ans, les autres musées comme les muséums ont aussi souvent été cités.

Bien entendu, le musée de science n'est pas le seul à jouer ce rôle et celui de l'école est déterminant, en particulier par la relation humaine. Ainsi, pour $70 \%$ des vocations scientifiques il y a eu « un jour, un prof ». Dans 60\% des cas, l'importance de la sensibilisation dès l'enfance est fondamentale, d'où l'intérêt d'enseigner les sciences de façon attractive et pratique dès la petite école et d'adapter les expositions aux jeunes publics.

La manière d'aborder les sciences est déterminante dans le développement de l'intérêt pour les sciences. En effet, on retrouve le même moteur pour tous: la curiosité et l'envie de comprendre. Et cela passe toujours par des situations d'observation ou de manipulations qui stimulent le raisonnement.

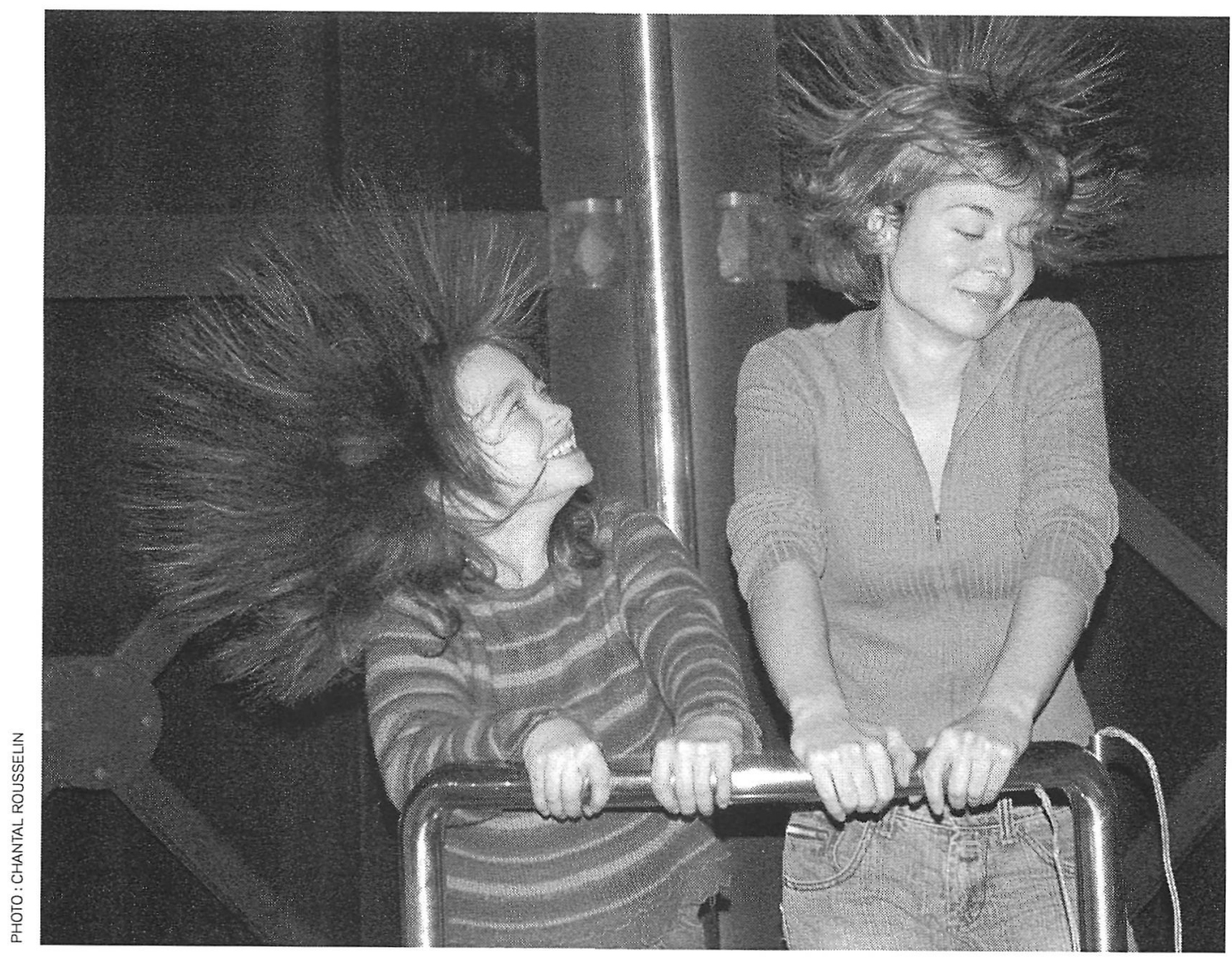

Exposé d'électrostatique au Palais de la découverte. 


\section{Un équilibre à trouver entre visites scolaires et visites de loisir}

Le musée n'est pas l'école et ne reçoit pas que des écoles. Par exemple, dans le cas du Palais de la découverte, $15 \%$ à $25 \%$ du public est représenté par les écoles avec un nombre de groupes scolaires assez stable (de 70000 à 80000 visiteurs/an). La proportion des élèves de l'école primaire (3 à 10 ans) varie en fonction des thèmes d'expositions temporaires, donc de leur lien avec les programmes scolaires.

Une enquête effectuée en 2006 montre que le public de loisir y représente en moyenne $80 \%$ de la fréquentation. Mais une proportion non négligeable de ce public correspond à des jeunes qui ont visité l'exposition avec leur classe et qui y reviennent en y entraînant leur parents, leurs frères et sœurs et leurs copains. Du côté du Palais de la découverte, la fidélisation des jeunes pendant plusieurs années est vérifiée. Les jeunes représentent $60 \%$ du public de fin de semaine et de vacances scolaires. Ils se répartissent comme suit:

- $20 \%$ enfants du primaire ( $26 \%$ à la Cité des sciences et de l'industrie (CSI) / Cité des enfants);

- $25 \%$ collégiens et lycéens (correspondant respectivement au Québec aux premier et second cycles du secondaire) (17\% à la CSI, y compris la médiathèque);

- $15 \%$ étudiants ( $18 \%$ à la CSI, y compris la médiathèque).

Cette même enquête dévoile que les personnes qui les ont incités à venir sont les parents (pour $76 \%$ des collégiens), les copains (38\% des lycéens) ou les amis ( $39 \%$ des étudiants). Par ailleurs, $32 \%$ de ces derniers veulent revoir ce qu'ils avaient vu lorsqu'ils étaient enfants.

La visite de loisir est souvent complémentaire à la visite scolaire. Les jeunes y apprécient de revoir ce qu'ils avaient vu avec la classe (pour 54\%), de le montrer à des copains (devenir le médiateur pour les pairs), d'avoir le temps de refaire les expériences et de voir d'autres démonstrations, de voir "en vrai» ce qu'ils ne connaissaient que par les schémas des livres (19\%), d'avoir le contact avec les chercheurs et les laboratoires de recherche. 


\section{Le contexte de la visite de loisir}

Au Palais de la découverte, les visites de loisir se déroulent dans un contexte différent selon l'âge des jeunes visiteurs. La visite des enfants de moins de 11 ans s'effectue avec les parents ou les grands-parents. Celle des jeunes de 12 à 14 ans (âge du collège ou premier cycle du secondaire au Québec) s'effectue à $76 \%$ avec les parents, sinon en groupe pour les plus âgés d'entre eux. Celle des jeunes de 15 à 18 ans (lycée ou deuxième cycle du secondaire) s'effectue souvent entre copains ou copines (38\%), en groupe de 2 à 6 jeunes, majoritairement du même sexe.

Chez les 18 à 24 ans, la visite a lieu surtout en couple, l'un des deux étant souvent non scientifique et l'autre voulant utiliser ce lieu pour lui montrer en quoi la science est intéressante et donc lui faire comprendre sa passion.

Nous avons cherché à savoir ce qui motive la visite des jeunes. Ce sont d'abord les manipulations interactives à faire soi-même qui attirent $48 \%$ des moins de 13 ans. Viennent ensuite les vraies expériences scientifiques (36\%). Chez les jeunes de plus de 14 ans, on constate un besoin d'authenticité, car ils privilégient d'abord les vraies expériences (pour $50 \%$ ) avant les manipulations interactives (38\%).

Pour tous les jeunes, quel que soit leur âge, la rencontre avec des médiateurs scientifiques est très importante et même considérée comme essentielle pour la moitié des plus de 14 ans. Ce dialogue avec des médiateurs est d'ailleurs plébiscité par les publics non scientifiques. Les expériences à faire soi-même, les vraies expériences et la présence de scientifiques sont donc les éléments fondamentaux à développer si l'on veut attirer les adolescents à l'âge de l'orientation.

Pour un lieu comme le Palais de la découverte, le développement des publics de loisir passe par le renforcement des publics des fins de semaine, en utilisant l'attraction des expositions temporaires pour un public de la région, ainsi que le public des mercredis en direction des grands-parents qui peuvent y conduire leurs petits-enfants (qui sont en congés alors que leurs parents travaillent). Les petites vacances (Toussaint, Noël, hiver, printemps) étant toujours des moments d'affluence en raison au couplage des publics franciliens et surtout de province, il faut développer la fréquentation d'été, en particulier en visant des publics étrangers. Pour attirer ces nouveaux publics de touristes étrangers non francophones, le Palais développe une offre muséographique avec des textes en trois langues, un programme d'exposés en anglais, des agents d'accueil bilingues, un dépliant de visite et des parcours de visite (papier et $\mathrm{MP}_{3}$ ) bilingues. Pour attirer les visiteurs étrangers, par ailleurs, nous développons des campagnes de communication à leur intention (Air-France, trains, hôtels et offices de tourisme). Ainsi à l'été 2008, nous avons eu la meilleure fréquentation jamais enregistrée. 


\section{Ce qui fait le succès des expositions scientifiques}

L'analyse des parcours de visite montre que les clés de succès auprès des publics scolaires et de loisirs sont l'équilibre entre des expositions temporaires attirant des non-scientifiques, prolongées par un parcours de visite qui inclut des expériences et des démonstrations spectaculaires.

Il est important de prendre en compte dès la conception le rapport à la science et au savoir des visiteurs. L'émotion, née de la surprise, développe l'intérêt et est conservée dans les souvenirs. Elle peut être créée en mettant la science en spectacle. Ces situations, comme l'expérience d'électrostatique où les cheveux se dressent sur la tête, marquent la mémoire en faisant passer de l'émerveillement au mystère dévoilé, grâce à l'accompagnement par des médiateurs scientifiques.

Un autre ingrédient du succès est le plaisir créé par des mises en situation qui suscitent l'étonnement, la surprise. Il est soutenu par l'attractivité, la recherche de la réussite dans les manipulations, le recours aux perceptions sensorielles. Les mises en situation doivent aussi favoriser les échanges entre les jeunes, par des aventures à partager entre copains, par la compétition entre visiteurs sur certaines manipulations. La mobilisation pour une activité par des jeux moteurs qui mettent à contribution l'adresse, la force, l'agilité, le mouvement, induisent la recherche de solutions, le déclenchement du questionnement, dont le rôle est aussi important pour la visite de loisir que pour la visite scolaire.

Ce contact avec le réel est la caractéristique incontournable d'un musée. Il n'y a que dans ces lieux que l'on peut voir en direct des objets authentiques, des expériences que l'on ne peut trouver ailleurs.

Une particularité du Palais de la découverte concerne la section "vivant" de l'exposition, surtout la présentation de petits animaux et d'insectes (fourmis, termites, grillons, araignées, une pieuvre, une école des rats...). Au Palais de la découverte, les espaces avec ces animaux focalisent l'attention des enfants et développent calme et sérénité.

Enfin la présence de scientifiques médiateurs qui font des expériences une spécificité du Palais de la découverte - est un élément essentiel du succès de ce musée, à la fois pour les jeunes et pour les publics non scientifiques qui apprécient l'adaptation du discours et les réponses aux questions qu'ils se posent. Ces publics sont captivés par la transmission de la passion des scientifiques et les formules d'exposés qui leur laissent le temps de comprendre. Une partie de ces médiateurs sont de jeunes chercheurs en préparation de thèse qui assurent des vacations et présentent au public la science en train de se faire, ce qui passionne les adolescents. 


\section{Les conditions d'un complément par rapport à l'école}

Une visite scolaire utile est une visite incluse dans une progression pédagogique : une visite préparée et exploitée en classe, encadrée par l'enseignant, même s'il s'appuie sur les médiateurs du musée.

Les dispositifs interactifs ont toujours du succès, mais pour que l'école puisse bien utiliser l'intérêt qu'ils déclenchent, il est essentiel que les savoirs soient contextualisés, d'où l'importance des regroupements thématiques et de la scénographie de l'exposition.

La présence d'un service pédagogique avec des enseignants de terrain déchargés à temps partiel y est capitale. Ils connaissent à la fois les besoins des enseignants et les spécificités du musée. Ils peuvent donc influencer certaines orientations et surtout, par leur connaissance privilégiée des expositions, assurer un accompagnement pédagogique, créer des documents d'aide à la visite et des parcours de visite. De plus, leur proximité avec la conception des expositions sur de nouvelles thématiques liées aux avancées de la science leur permet de créer des manipulations qui pourront ensuite passer du musée à l'école, ce qui n'est pas sans impact sur l'évolution des programmes scolaires.

De plus ils peuvent assurer la formation indispensable des enseignants à l'utilisation des musées. L'exemple de la formation initiale des enseignants du primaire, dont la majorité n'est pas scientifique, est exemplaire. Le musée de science, en montrant les sciences de façon attractive, permet de développer l'intérêt des enseignants non scientifiques pour les sciences. En leur donnant loccasion d'observer des enfants dans des démarches de découverte active des sciences, il leur en démontre l'intérêt pédagogique en classe.

En conclusion, pour que loisirs et éducation fassent bon ménage, il faut à la fois offrir des expositions attractives et éducatives, des expositions temporaires aux sujets évocateurs et attractifs, des expositions permanentes complémentaires de l'école, un billet unique et des parcours qui conduisent des expositions temporaires aux permanentes et, surtout, une médiation humaine qui s'adapte aux différents publics. 


\section{Summary}

[Translated by Allana Carlyle]

The context and objectives of a school lesson compared to those of a recreational visit to a science museum are very different, or even opposite. And yet, it is possible to join these two learning strategies in one location with the common goals being the development of young people's interest towards the sciences, the creation of educational supports for teachers and the organization of field trips. This has been the challenge of the Palais de la découverte in Paris for the last 70 years, and is still continuing today.

Scientific exhibitions allow children to become immersed in science through the use of experiments and spectacular objects that trigger emotions and mark their memories forever. They can bring to life opportunities for discovery which evoke questioning. They can present experiments and other phenomena in true grandeur, which students do not otherwise experience other than through diagrams in books. As for schools, they have the ability to introduce a longer-term investigative approach to learning science. Such achievement is only possible if the museum proposes a structured approach on themes that interest the school and if the teachers integrate the field trip visit into the teaching process.

These assets are used at the Palais de la découverte through the presence of parttime teachers responsible for the museum's educational programming and by the omnipresence of scientific mediators who carry out scientific experiments in front of the children. This dialogue with scientists who share their passion and appropriately adapt their lectures is equally appreciated by children and adolescents. It is also a teaching strategy for elementary school teachers who are rarely science experts.

The result is 70 years of scientific vocational development for children! 